\title{
Intraoperative Bildgebung in der Veterinärmedizin
}

\author{
Andrea Meyer-Lindenberg, Tim Kilchling
}

\section{Zusammenfassung}

Anlehnend an die Humanmedizin spielt auch in der Veterinärmedizin die intraoperative Bildgebung, insbesondere bei orthopädischen Eingriffen, eine große Rolle. Als häufigstes Medium kommt im Operationssaal dabei der C-Bogen zum Einsatz, der basierend auf der Röntgentechnik intra operationem Durchleuchtungsaufnahmen von Knochen und Implantaten anfertigt, die dem Chirurgen die Arbeit sehr erleichtern. Die Anwendung verkürzt durch die direkt in der Operation mögliche Evaluierung der Wiederherstellung der anatomischen Reposition der Knochen bzw. des korrekten Sitzes der Implantate die Operationszeit und damit die Narkosedauer. Zudem wird dadurch die Zahl der Revisionsoperationen deutlich verringert. Durch den Einsatz von Kontrastmittel ist die intraoperative Bildgebung mithilfe des $\mathrm{C}$ Bogens auch in der Kardiologie und Weichteilchirurgie, insbesondere im Rahmen von minimalinvasiven Interventionen, nicht mehr wegzudenken. In dieser Arbeit werden einige Einsatzbereiche des C-Bogens anhand von Beispielen beschrieben. Des Weiteren werden andere intraoperative Bildgebungstechniken, die in der Veterinärmedizin angewendet werden, wie der Ultraschall, die Computer- und Magnetresonanztomografie, kurz beschrieben.

\section{Einleitung}

In der sich in den letzten Jahrzehnten stark im Wandel befindlichen Veterinärmedizin rückt die Diagnostik und Thera-

OP-JOURNAL 2014; 30: 178-187

(c) Georg Thieme Verlag KG Stuttgart · New York DOI http://dx.doi.org/10.1055/s-0034-1383269

\section{Intraoperative Diagnostic Imaging in Veterinary Medicine}

With reference to human medicine intraoperative diagnostic imaging also plays a major role in veterinary medicine, especially in orthopedic surgery. As the most frequent medium the $\mathrm{C}$-arm is used in the operating room, which, based on the X-ray technology, provides intraoperative radiographic snapshots of bones and implants which greatly facilitates the surgeon's work. The application of the C-arm shortens the operation time as well as the duration of anesthesia due to providing the possibility of evaluating directly during the operation, e.g. the restoration of the anatomic reposition of the bones or the correct position of the implants. Moreover, using this medium, the number of revision operations is significantly reduced. By the application of contrast medium, intraoperative diagnostic imaging by using the C-arm has become indispensable also in the soft tissue surgery and cardiology, in particular in the field of minimally invasive interventions. Some fields of application of the C-arm, based on examples, are described in this article. Further, other intraoperative imaging techniques such as ultrasound, computed tomography and magnetic resonance imaging which are applied in veterinary medicine are shortly described. lisierten Operationstechniken bereits Einzug in die Operationssäle insbesondere größerer Kliniken gehalten und damit verbunden natürlich auch die verschiedensten Verfahren und Techniken zur Diagnostik und Operationsplanung. Wie in der Humanmedizin hat sich die intraoperative Bildgebung inzwischen auch in der Veterinärmedizin etabliert. In diesem Überblick werden gängige Operationen, bei denen die intraoperative Bildgebung angewendet wird, dargestellt und ein Ausblick über mögliche Zukunftsperspektiven in der Veterinärmedizin gegeben.

\section{Intraoperative Bildgebung mittels C-Bogen}

Der C-Bogen ist ein mobiles, auf dem Prinzip der Röntgentechnologie arbeitendes, bildgebendes Gerät, bei dem die Röntgenquelle und der Röntgendetektor fest miteinander durch einen C-förmigen Arm verbunden sind. Die Technologie des C-Bogens hat sich seit seiner Einführung 1955 rasant weiterentwickelt. Hochauflösende Röntgenbilder in Echtzeit werden durch das Senden von Röntgenstrahlen aus dem Generator (Röntgenquelle) erzeugt, die nach dem Durchdringen des Körpers von dem Detektor oder Bildverstärker umgewandelt werden.

Die Beweglichkeit des Generators und der Röntgenquelle in allen 3 Ebenen ermöglicht Momentaufnahmen von allen erwünschten Positionen und erleichtert, insbesondere bei schwierigen Frakturen durch die Kontrolle der Reposition der Fragmente und die Positionierung der Implantate, die Arbeit des Chirurgen.

pie von kleinen Haus- und Heimtieren, insbesondere Hunden und Katzen, immer mehr in den Vordergrund. Durch die stetig steigenden Ansprüche der Tierbesitzer an den Tierarzt in seinem Fachgebiet der Veterinärmedizin entwickeln sich die diagnostischen Verfahren und therapeutischen Möglichkeiten immer weiter. Inzwischen haben die unterschiedlichsten, teilweise hoch spezia- 


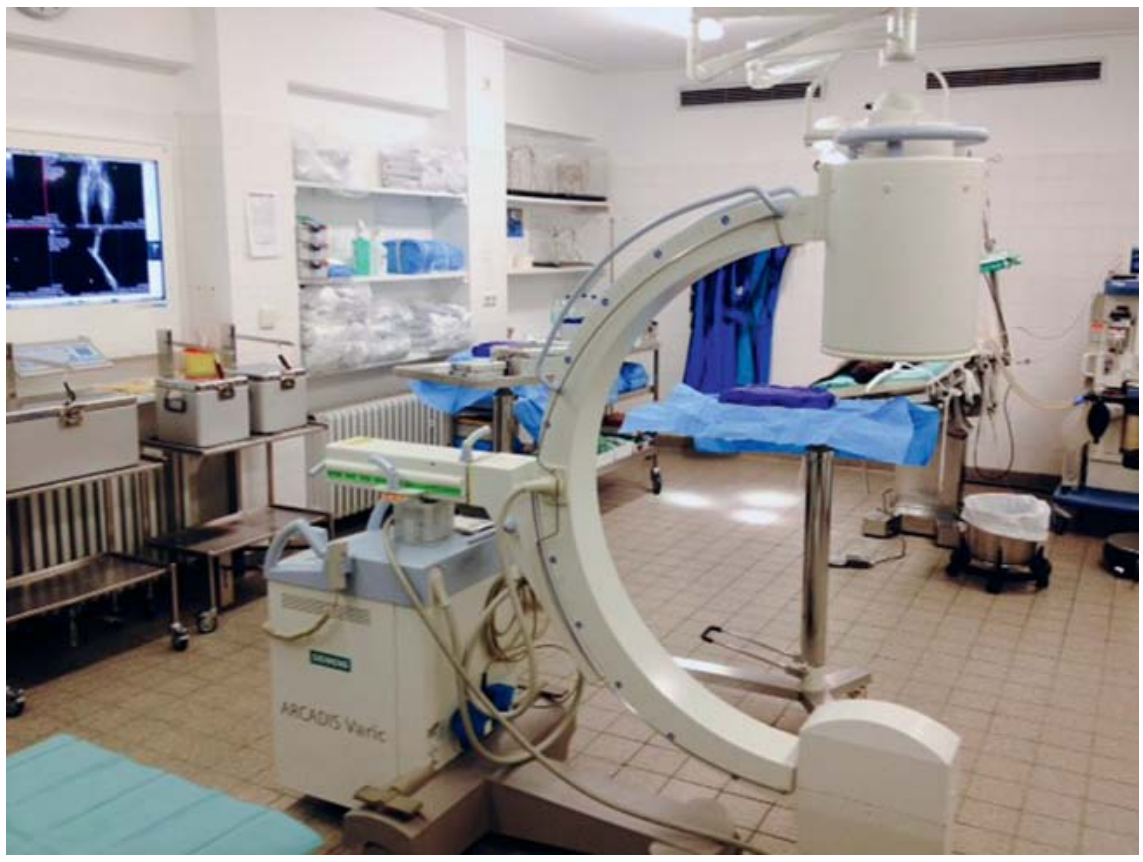

Abb. 1 C-Bogen (Siemens ARCADIS Varic) im Operationssaal der Chirurgischen und Gynäkologischen Kleintierklinik der LMU München.

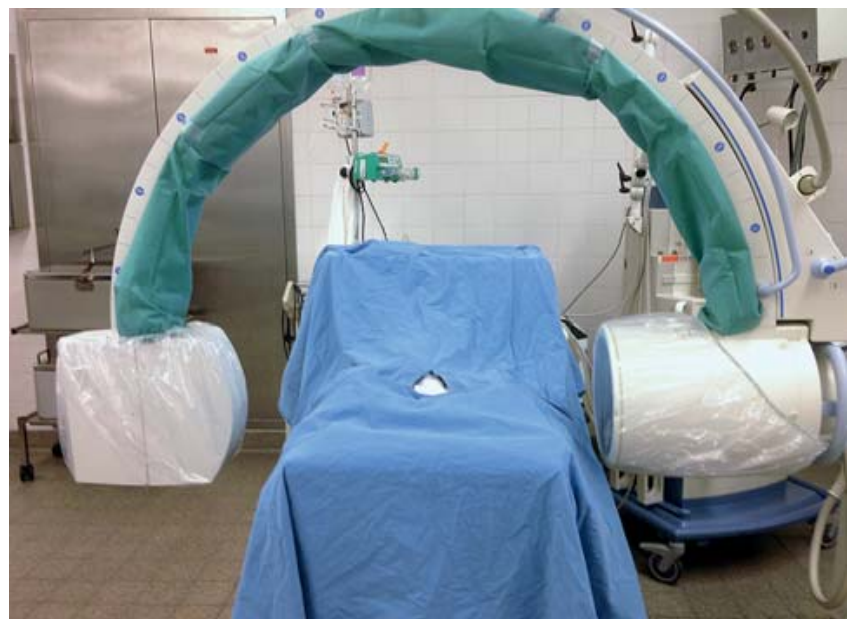

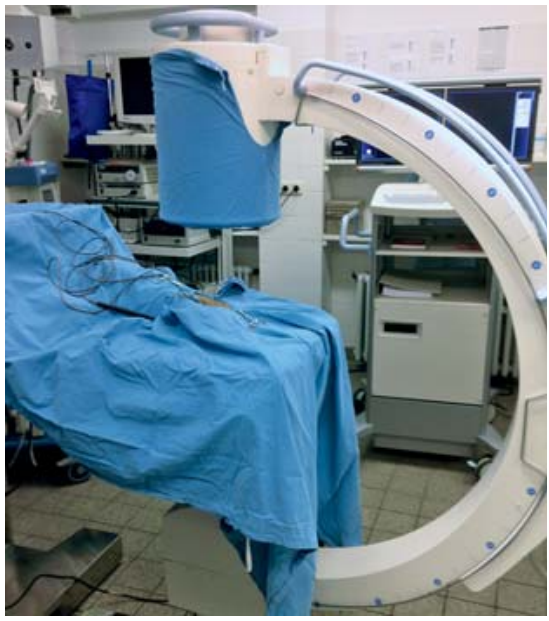

Abb. 2 Für die einfache Durchleuchtung in 1 Ebene ist der Bildverstärker des C-Bogens mit einem Stoffüberzug abgedeckt.

die Sterilität berücksichtigt werden. Aus diesem Grunde ist es erforderlich, je nach Einsatzgebiet und Ausmaß der Verwendung entweder nur den Bildverstärker (Abb. 2) oder auch die Röntgenröhre und den C-Arm durch speziell dafür angefertigte sterile Stoff- oder Plastiküberzüge abzudecken.

Insbesondere, wenn der C-Arm „über Kopf" angewendet wird, um z.B. während der Durchleuchtung ein Implantat korrekt zu setzen, müssen der Bildverstärker und die Röntgenröhre inkl. des C-Armes steril abgedeckt werden (Abb. 3). Somit kann dann der C-Bogen in beliebigen Positionen über das Operationsfeld gefahren und die entsprechenden Bilder angefertigt werden.

Nach jeder Operation sollte das Durchleuchtungsgerät mit einem DGHM-gelisteten Scheuer-Wisch-Desinfektionsmittel wischdesinfiziert werden. Nicht benutzte Geräte sollten 1-mal wöchentlich gereinigt werden. Wird das Gerät länger nicht genutzt, sollte die Durchleuchtungsanlage mit Tüchern zum Staubschutz abgedeckt werden [2].

知 reichen eingesetzt. Im Vordergrund steht hier die Anwendung bei Patienten mit orthopädischen Operationen und in der Traumatologie.

Aber auch in anderen Bereichen, wie z.B. Gastroenterologie, Urologie oder Kardiologie (unter Verwendung von Kontrastmittel) sowie verschiedenen Weichteiloperationen hat sich der Einsatz des C-Bogens in der Veterinärchirurgie als essenziell und sehr hilfreich erwiesen. Bei seinem Einsatz während der verschiedenen Operationen muss immer
Bei der Verwendung eines C-Bogens sind die Strahlenschutzaspekte zu berücksichtigen.

Für den Schutz von Personen, die ständig oder gelegentlich Röntgenstrahlung ausgesetzt sind, wurde eine Reihe von Gesetzen und Verordnungen erlassen. Hierzu zählen die Röntgenverordnung, die Strahlenschutzverordnung, das Atomgesetz u.a., in denen geregelt ist, wie hoch die Strahlenbelastung und die Höchst- 
werte einer Person sein dürfen. Maßnahmen zum Schutz vor Strahlung sind auch für die Veterinärmedizin verpflichtend. Der Kontrollbereich des C-Bogens wird mit wenigen Metern Radius angegeben. Innerhalb dieses Bereichs ist das Tragen einer Bleischürze und eines Dosimeters unter der Röntgenschürze vorgeschrieben. Der Operateur und seine Assistenten tragen bereits bevor sie sich für die Operation einwaschen, eine Röntgenschürze und einen Schilddrüsenschutz, über die dann der sterile OP-Mantel gezogen wird. Weitere Personen, die sich im Operationssaal aufhalten, dies betrifft bspw. den Anästhesisten, aber v.a. auch das Personal, das den C-Bogen bedient, müssen für die Durchleuchtungsphasen ebenfalls eine Röntgenschürze und Schilddrüsenschutz tragen. Alle anderen Personen, die nicht zwingend für diese Zeitspanne im Operationssaal erforderlich sind, wie z. B. Assistenten oder Studenten, müssen aus Strahlenschutzgründen den Operationssaal für den Moment der Durchleuchtung verlassen. Bei Operationen, bei denen über einen längeren Zeitabschnitt durchleuchtet wird, bspw. bei Eingriffen am Herzen, sollte zusätzlich zu Röntgenschürze und Schilddrüsenschutz eine Bleibrille getragen werden.

Das Personal, das den C-Bogen bedient, muss den technischen Umgang mit dem Gerät sicher beherrschen.

Nach erfolgter Einarbeitung und Strahlenschutzeinweisung empfiehlt sich auch, regelmäßige Trockenübungen mit dem den Durchleuchter bedienenden Personal durchzuführen, um den sicheren und präzisen Umgang mit dem Schwenkarm zu trainieren. Ein sicherer Umgang und Kenntnisse des Geräts erleichtern die Durchleuchtung im Routine- und im Ernstfall, auch im Notdienst, und ermöglichen ein zeitsparendes Arbeiten im Operationssaal.

Neben der 2-D-Bildgebung im Operationssaal beschreiben Wilde et al. [3] die Vorteile der intraoperativen 3-D-Bildgebung mit einem speziell dafür konzipierten C-Bogen bei der chirurgischen Versorgung intraartikulärer Frakturen des Sprunggelenks in der Humanmedizin. Hierbei wurde nach der Reposition die Fraktur osteosynthetisch versorgt mithilfe von konventioneller 2-D-Bildgebung. Anschließend wurde vor Wundverschluss mittels eines hochauflösenden multiplanaren 3-D-Bilddatensatzes ein Bild aus 100 Einzeldurchleuchtungen generiert, die im Rahmen einer 1-minütigen Rotation um das Untersuchungsgebiet akquiriert wurden. Falls dadurch eine Korrektur indiziert war, konnte diese sogleich durchgeführt werden. Die 3-D-Bildgebung wird für die Humanmedizin als sicheres und zuverlässiges Verfahren zur intraoperativen Evaluierung osteosynthetischer Versorgungen beschrieben. Durch die Identifikation von Fehlstellungen oder Fehllagen von Implantaten ist es möglich, bereits gleich im OP eine sofortige Revision bzw. Korrektur durchzuführen. Ein kleiner Nachteil ist eine geringfügig längere Operationsdauer, jedoch kann durch dieses Verfahren ein strahlenintensiveres postoperatives CT ersetzt werden [3]. Auch andere Autoren beschreiben die Vorteile der 3-D-Bildgebung [4].

Der Vorteil der intraoperativen 3-D-Bildgebung wird ebenfalls in einer Arbeit von Richter [5] konstatiert, in der dieses Verfahren insbesondere bei der chirurgischen Versorgung von Fuß- und Sprunggelenkverletzungen zum Einsatz kommt. Auswertungen seiner Untersuchungen zeigten, dass in gut einem Drittel der Fälle die intraoperative 3-D-Bildgebung eine nicht optimale Knochen- und/oder Implantatposition nachwies, die durch die konventionelle 2-D-Bildgebung nicht erkennbar gewesen wäre und im selben Eingriff dank der 3-D-Darstellung korrigiert werden konnte. Das führte letztendlich zu einer niedrigeren Anzahl von Korrekturoperationen. In der Veterinärmedizin ist diese hochauflösende Technik noch nicht sehr weit verbreitet, sondern hier dominiert die intraoperative 2-D-Bildgebung. Nachfolgend sollen einige Anwendungsbeispiele für die intraoperative Bildgebung mittels C-Bogen in der Chirurgischen und Gynäkologischen Kleintierklinik der Ludwig-MaximiliansUniversität München dargestellt werden.

\section{Einsatz in der Orthopädie und Traumatologie}

In der Orthopädie und Traumatologie wird der C-Bogen im Rahmen der intraoperativen Bildgebung sowohl für viele Routineoperationen als auch für minimalinvasive Eingriffe, wie bspw. zur Kontrolle der Reposition von Fragmenten, genutzt.

$\mathrm{Zu}$ den Routineoperationen gehört z.B. der vordere Kreuzbandriss, der insbesondere beim Hund, aber auch bei Katzen, mithilfe der Tibial Plateau Leveling Osteotomy (TPLO) versorgt werden kann. Hier wird durch eine Umstellungsosteotomie des proximalen Tibiaplateaus der Tibiaplateauwinkel, der zwischen 20 und $30^{\circ}$ liegt, auf ca. $6^{\circ}$ reduziert, was eine Umverteilung der Scherkräfte im Kniegelenk zur Folge hat. Mit dieser Umstellung wird der kraniale Schub des Femurs auf die Tibia, der normalerweise vom intakten vorderen Kreuzband aufgefangen wird, aufgehoben. Im Rahmen der Operation wird eine kreis- bzw. sichelförmige Osteotomie der proximalen Tibia vorgenommen und das Tibiaplateau um die zuvor berechnete Strecke rotiert und anschließend mit einer speziellen TPLO-Platte an der distalen Tibia fixiert [6]. Für diese Fixierung stehen sowohl winkelstabile als auch herkömmliche Platten verschiedener Hersteller zur Verfügung. Wird ein nicht winkelstabiles System verwendet, so kann es leicht passieren, dass eine der proximalen Schrauben in den Gelenkspalt des Kniegelenks penetriert. Dies ist ein häufiger Fehler bei der Implantation von TPLO-Platten und kann durch den Einsatz der intraoperativen Bildgebung mittels C-Bogen leicht verhindert werden. Dabei kann die Richtung mithilfe des Bildwandlers während des Bohrens des Schraubenlochs kontrolliert werden, was mit einer relativen Strahlenbelastung für den Chirurgen einhergeht. In der Regel erfolgt die Kontrolle jedoch nach der Schraubenimplantation. Im Falle einer Fehlpositionierung kann sie dann noch intraoperativ durch eine kürzere Schraube ausgetauscht oder das Bohrloch neu gesetzt werden. Bei den meisten winkelstabilen Platten sind die Positionierung und die Winkelung und damit die Richtung der Schrauben bereits vorgegeben, sodass die Schrauben nur bei falsch positionierter Platte in den Gelenkspalt gelangen können. Aber auch hier ist die intraoperative Überprüfung des Sitzes der Platte und der Länge der Schrauben mithilfe des C-Bogens hilfreich.

Durch den schwenkbaren Arm des C-Bogens lassen sich so hervorragend Darstellungen in verschiedenen Ebenen anfertigen (Abb. 4).

Eine weitere Indikation stellt in der Orthopädie die Endoprothetik dar. Der Einsatz von Hüftendoprothesen ist beim Hund heutzutage eine Routinetherapie z. B. bei Vorliegen einer Hüftgelenksdysplasie, die insbesondere bei mittelgroßen und großen Hunde noch immer relativ häufig vorkommt. Aber auch traumatische Insulte, wie z.B. eine Hüftgelenks- 


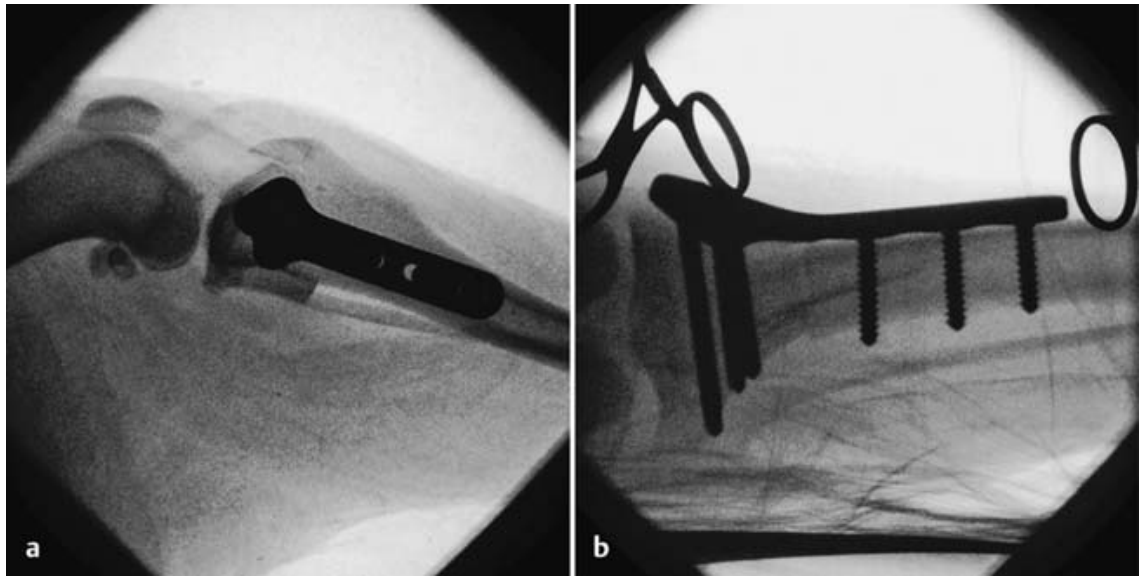

Abb.4a und b Intraoperative Bildgebung mittels C-Bogen zur Überprüfung der Lage einer TPLO-Platte, der Rotation des proximalen Tibiaplateaus und der Länge der verwendeten Schrauben im mediolateralen (a) und kraniokaudalen (b) Strahlengang.
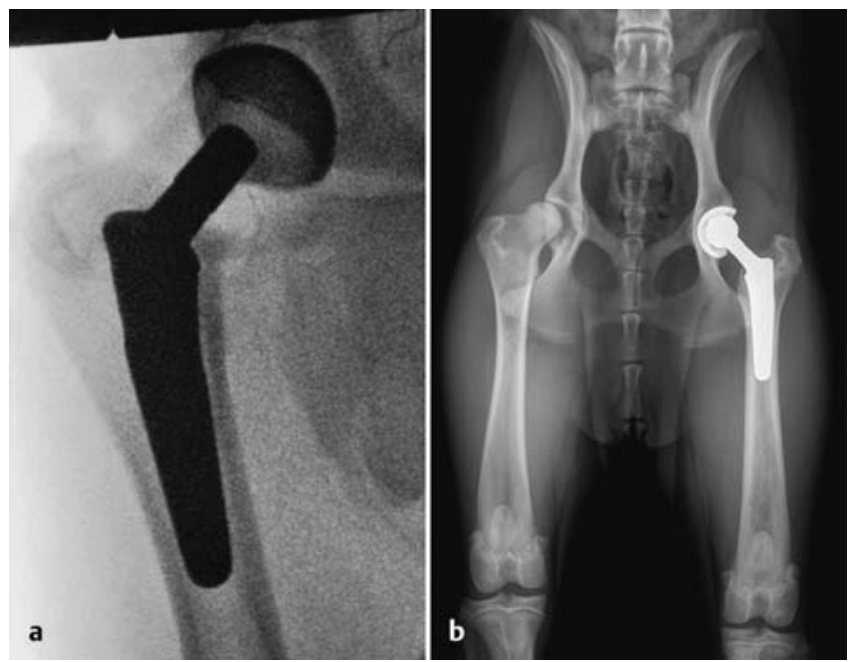

Abb. 5 a und $b$ Intraoperative Darstellung des proximalen Femurschafts mit Implantat bei einer nicht zementierten Endoprothese (Fa. Biomedtrix, Boonton, USA) unter Kontrolle mit Durchleuchtung (a) und (b) postoperatives Röntgenbild.

luxation oder nicht adäquat versorgte/ verheilte Beckenfrakturen können eine Hüftgelenksendoprothese erforderlich machen. Zum Einsatz kommen verschiedene Systeme unterschiedlicher Hersteller, wobei hier im Wesentlichen die ze- mentierten von den nicht zementierten Prothesensystemen zu unterscheiden sind. Die Planung der Operation und die der Auswahl der Größe der Endoprothese (Schaft/Pfanne) erfolgt immer anhand der präoperativ angefertigten Röntgen- aufnahmen in 2 Ebenen, wobei die Planung entweder anhand von speziellen Schablonen erfolgt oder, bei digitaler Bildgebung, anhand eines speziellen Softwareprogramms (z.B. in der Chirurgischen und Gynäkologischen Kleintierklinik: Dicom PACS, Fa. Oehm und Rehbein, Rostock).

Jedoch kann es hier, insbesondere bei den nicht zementierten Hüftendoprothesen, die sehr passgenau implantiert werden müssen, hilfreich sein, den korrekten Sitz und die Passgenauigkeit der Schaftkomponente mithilfe der intraoperativen Bildgebung zu überprüfen (Abb. 5).

Ein weiterer Einsatz des C-Bogens in der Orthopädie ist die chirurgische Versorgung eines isolierten Processus anconaeus (IPA) der Ulna. Dabei handelt es sich um eine Erkrankung, die zur Ellbogengelenksdysplasie des Hundes gerechnet wird. Sie tritt bei bestimmten mittelgroßen und großwüchsigen Rassen, wie bspw. dem Schäferhund, besonders häufig auf und ist erblich bedingt. Es handelt sich dabei um eine Störung der enchondralen Ossifikation im Bereich der bei diesen Rassen vorliegenden Wachstumsfuge des Processus anconaeus. Normalerweise fusioniert der Processus anconaeus um den 5 . Lebensmonat herum mit der Ulna. Kommt es zu keiner Fusion, liegt die Erkrankung eines IPA vor. Obwohl es sich nur um einen relativ kleinen Knochenfortsatz handelt, schreibt man dem Processus anconaeus eine wichtige Funktion zu, und zwar die Stabilisierung des Ellbogengelenks während der Streckung. Daher wird der Erhalt des Processus anconoaeus angestrebt, da es sonst häufig zur Ausbildung einer Osteoarthrose im Ellbogengelenk kommt mit
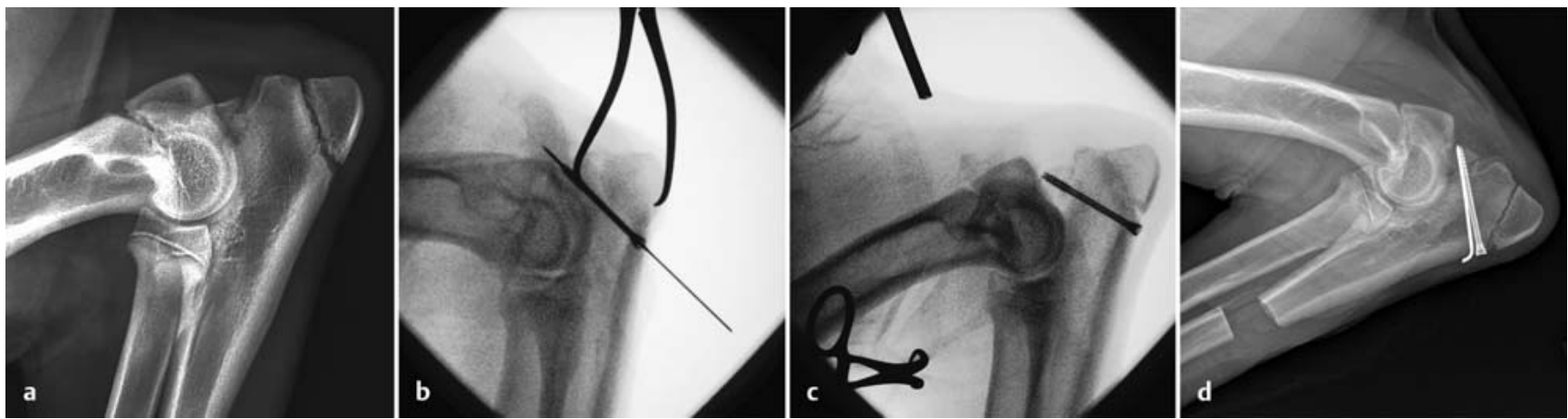

Abb. 6 a bis d Röntgenaufnahme eines Deutschen Schäferhunds mit isoliertem Processus anconaeus in mediolateralem Strahlengang (a). Intraoperative Bildgebung bei der Fixation eines isolierten Proc. anconaeus: Die Reposition erfolgt mittels einer Zweipunktzange und die Vorfixation mit einem Kirschner-Bohrdraht (b). Für die endgültige Fixation wird eine kanülierte Schraube über den Bohrdraht eingesetzt, die als Zugschraube fungiert, und der Bohrdraht wieder entfernt (c). Zusätzlich wird zur Rotationsstabilität ein weiterer Bohrdraht eingesetzt und eine Osteotomie der Ulna durchgeführt. Postoperativ erfolgt eine Röntgenkontrolle (d). 
einhergehender Lahmheit [7]. Die Fixation des kleinen Knochenstücks ist aufgrund der geringen Größe nicht ganz einfach. Zwar kann der zu versorgende Bereich intra operationem recht gut eingesehen und zusätzlich zur korrekten Positionierung der Implantate ein Zielgerät zur Hilfe genommen werden, jedoch ist die intraoperative Bildgebung hilfreich, um die Richtung der Schraube besser zu bestimmen. Nach Reposition des Fragments und Adaptation mittels Zweipunktzange, erfolgt das Anlegen eines Bohrkanals und die Fixation des IPA mittels einer Zugschraube. Alternativ kann zunächst auch eine Vorfixation mithilfe eines Kirschner-Bohrdrahtes unter fluoroskopischer Kontrolle erfolgen, über den dann eine spezielle kanülierte Schraube in entsprechender Länge als Zugschraube eingesetzt wird (Abb.6). Zur Rotationsstabilität wird dann noch ein weiterer Kirschner-Bohrdraht eingesetzt.

Sehr hilfreich ist der C-Bogen auch zur intraoperativen Identifikation von Wachstumsfugen.

So kann es beim Hund, selten bei der Katze, nötig sein, eine Symphysiodese vorzunehmen. Dies kann insbesondere bei Wachstumsstörungen im Bereich der Vordergliedmaße notwendig sein, wenn z.B. die distale Epiphysenfuge des Radius partiell geschädigt ist und nur ein Teil der Fuge eine Wachstumspotenz aufweist. Oft ist in diesen Fällen gleichzeitig auch die distale Epiphysenfuge der Ulna, z. B. durch ein Trauma oder einen persistierenden Knorpelzapfen, geschädigt, sodass es während des Wachstums zu einem Radius curvus mit Carpus valgus gekommen ist. Dies kann bei einem noch wachsenden Hund in Abhängigkeit vom Zeitpunkt des Auftretens mithilfe einer Ulnaosteomie mit partieller Epiphysiodese behandelt werden. Meistens ist hier der laterale Anteil der distalen Radiusepiphysenfuge betroffen, sodass hier der mediale Anteil am Wachstum gehindert werden muss. Das ist mit verschiedenen Techniken möglich, wie z.B. einer partiellen Epiphysiodese mittels spezieller Krampen. Um die richtige Position dieser Implantate zu bestimmen und sie korrekt über der Fuge zu platzieren, ist die intraoperative Bildgebung sehr hilfreich (Abb. 7).

In der Traumatologie findet der C-Bogen Anwendung bei Frakturen verschiedenster Art. So kann insbesondere bei komplizierten Gelenkfrakturen, wie z. B. Aze-

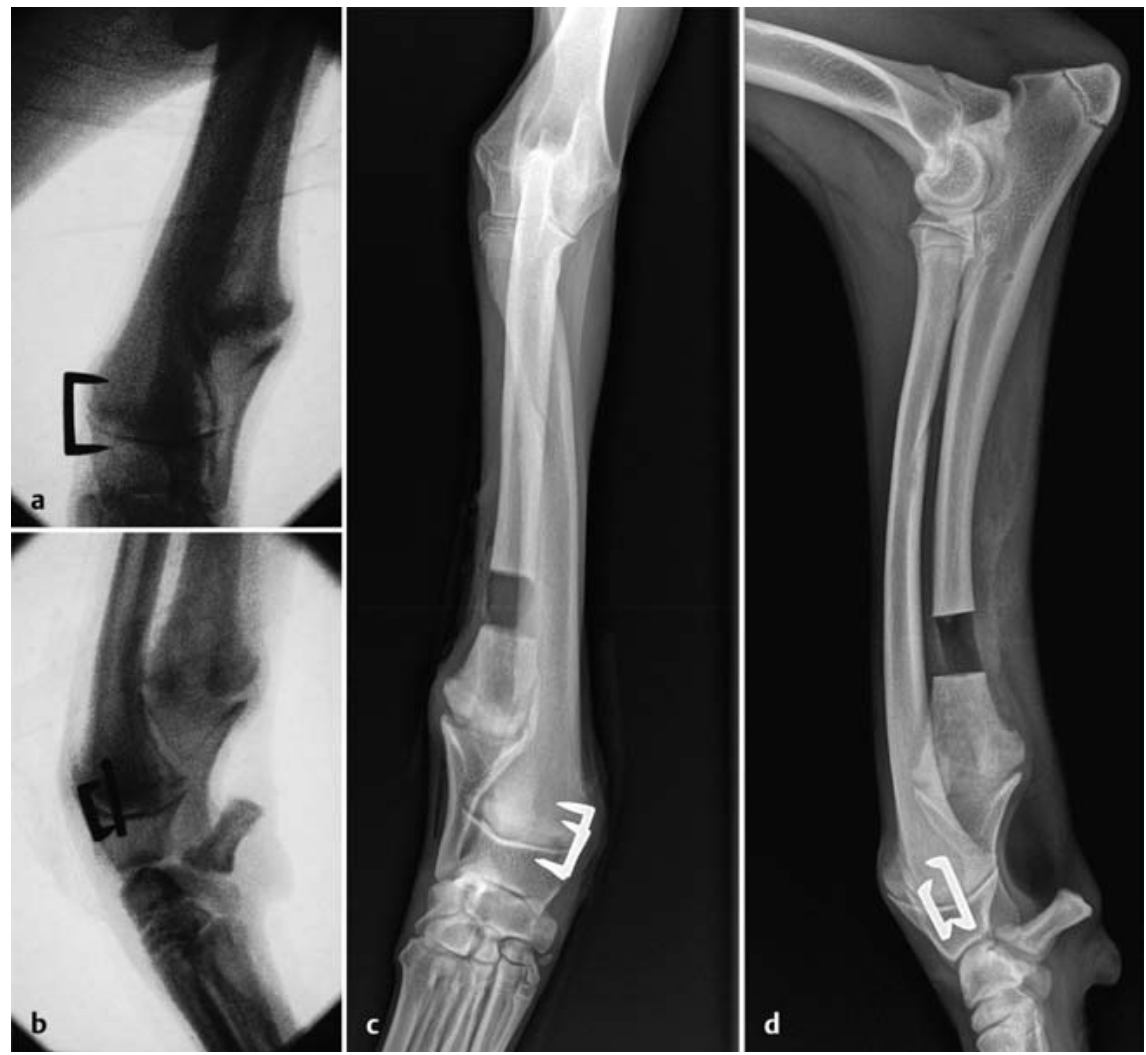

Abb.7a bis d Intraoperative Darstellung der distalen Radiusepiphysenfuge mittels C-Bogen. Hier kann die Position der Fuge genau lokalisiert und das Implantat exakt positioniert werden. Die postoperative Kontrolle erfolgt zusätzlich durch postoperative Röntgenaufnahmen im kraniokaudalen (c) und mediolateralen (d) Strahlengang. Zusätzlich zur partiellen Epiphysiodese der distalen Radiusfuge ist hier noch eine Ostektomie der Ulna vorgenommen worden.

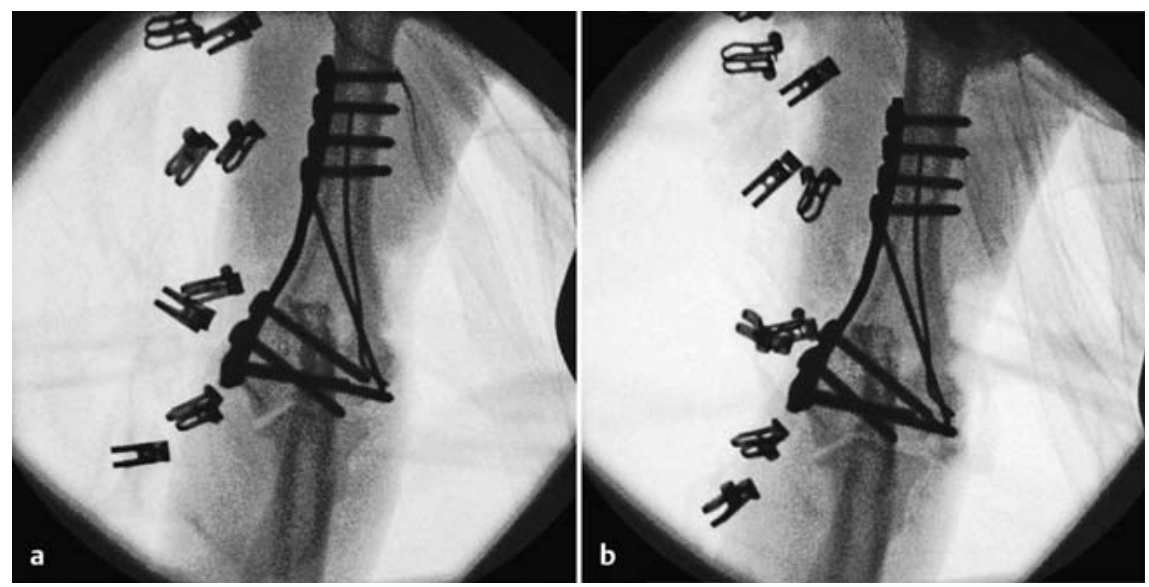

Abb. 8a und b Intraoperative Darstellung der Versorgung einer suprakondylären Fraktur des Condylus humeri. Die 2. Schraube von distal wurde zunächst etwas zu lang gewählt und ragte über die Gelenkfläche in den Radius hinein (a) und wurde noch intra operationem durch eine kürzere Schraube (b) ersetzt.

tabulumfrakturen oder Humerusfrakturen, bei denen die Gelenkfläche intra operationem nicht vollständig einzusehen ist, die anatomisch korrekte Reposition sofort überprüft und dann mit entsprechenden Implantaten zunächst ggf. vorfixiert und anschließend endgültig stabilisiert werden.
Besonders bei Gelenkfrakturen ist die exakte Reposition der Fragmente besonders wichtig, um die Gelenkflächen stufenfrei zu adaptieren. Nur so können die Gelenkfunktionalität vollständig wiederhergestellt und sekundäre Arthrosen vermieden werden. Auch der Sitz der Implantate kann mithilfe der intraoperati- 

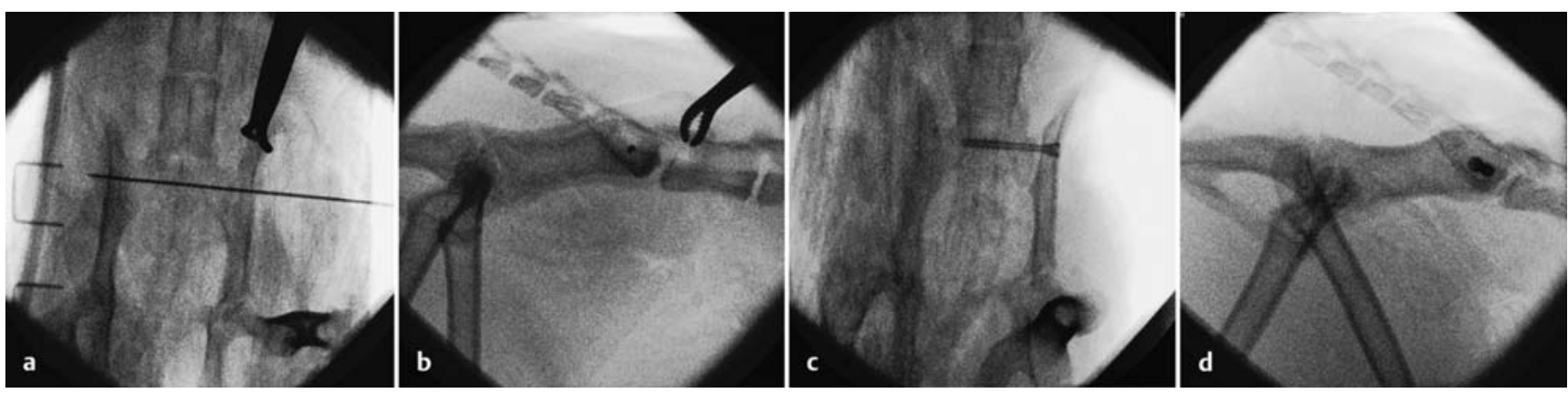

Abb. 9a bis d Intraoperative ventrodorsale (a) und laterolaterale (b) Darstellung eines Katzenbeckens mit bereits reponierter einseitiger Diastase (Positionszange oben bzw. kranial am Os ilium fixiert) und einem Kirschner-Bohrdraht, der durch das llium in das Kreuzbein eingebracht wurde. Die Durchleuchtung nach Implantation der kanülierten Schraube zeigt die korrekt gewählte Länge und Position der Schraube in 2 Ebenen (c, d).
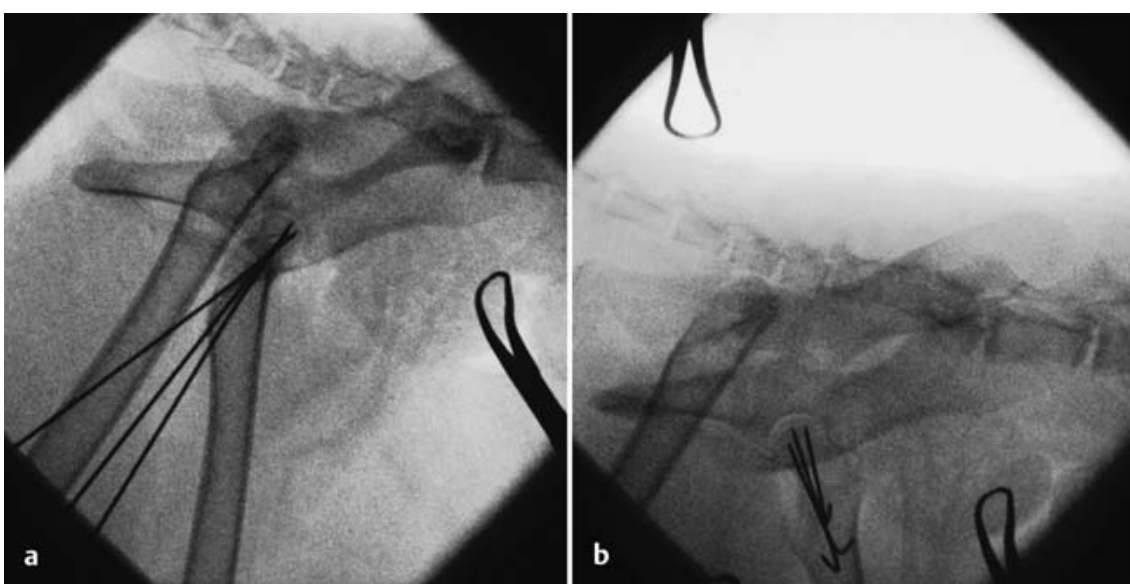

Abb. 10a und b intraoperative Bildgebung beim Setzen der Kirschner-Bohrdrähte vom Trochanter major aus in den Femurhals und Femurkopf (a); intraoperatives Durchleuchtungsbild nach endgültiger Positionierung und Umbiegung der Kirschner-Bohrdrähte; keiner der Pins ragt in das Gelenk hinein (b).

ven Bildgebung optimal überprüft und z.B. zu lang gewählte Implantate noch im Rahmen der Operation ausgetauscht werden (Abb. 8).

Aber auch bei einfachen Gelenkfrakturen wie z.B. Frakturen des lateralen Humeruskondylus kann der C-Bogen sehr hilfreich sein. So können diese Frakturen minimalinvasiv und unter fluoroskopischer Kontrolle reponiert und anschließend fixiert werden. So versorgten Cook et al. [8] in einer prospektiven klinischen Studie 10 Hunde mit Fraktur des lateralen Condylus humeri durch geschlossene Reposition und anschließender Fixation mittels interkondylärer Schraube und Kirschner-Bohrdraht unter fluoroskopischer Kontrolle mittels C-Bogen. In den meisten Fällen gelang ihnen eine nahezu stufenlose Reposition und sie konnten in allen Fällen eine vollständige Wiederherstellung der Gliedmaßenfunktion erreichen.

Auch bei anderen Frakturen und Luxationen wird die intraoperative Bild- gebung in der Veterinärmedizin beschrieben. So wird die Anwendung des C-Bogens zur Stabilisierung von iliosakralen Luxationsfrakturen bei 5 Hunden mittels transiliosakralem KirschnerBohrdraht beschrieben, der minimalinvasiv unter Durchleuchtungskontrolle korrekt platziert wird [9].

Bei traumatisch bedingten iliosakralen Luxationsfrakturen (Diastasen) bei der Katze ist die intraoperative Bildgebung zur Reposition und Positionierung der Implantate ebenfalls sehr hilfreich.

Insbesondere bei der Katze ist das Kreuzbein sehr klein und für die Positionierung von Schrauben bietet es nicht viel Platz. Da bei Katzen im Vergleich zum Hund sehr viel häufiger beidseitige Diastasen zusammen mit anderen Beckenfrakturen vorkommen, müssen meist beide Darmbeinanteile fixiert und damit 2 Schrauben in das Kreuzbein eingebracht werden. Gerade in diesen Fällen wird die intraoperative fluoroskopische Kontrolle zur Positionierung eingesetzt.
Bewährt haben sich für diesen Zweck kanülierte Schrauben (Fa. Z-Medical [Tuttlingen]; Fa. Synthes [Umkirch]), die aufgrund des gegenläufigen Gewindes am Schraubenkopf und am Schraubenende beim Eindrehen des Kopfes einen Zug auf das gefasste Fragment bewirken und somit als Zugschraube fungieren. Die Vorfixation erfolgt nach Reposition zunächst mit einem 1,0 mm dicken Kirschner-Bohrdraht durch das Darmbein ins Kreuzbein, über den dann nach Kontrolle des korrekten Sitzes (Abb. 9) die Kompressionsschraube eingebracht wird. Der Pin wird anschließend wieder entfernt und der korrekte Sitz und die Länge der Schraube fluoroskopisch kontrolliert (Abb. 9).

Auch bei der chirurgischen Versorgung von Femurkopf- oder -halsfrakturen bietet der intraoperative Einsatz des C-Bogens Vorteile, auch wenn es keine die Gelenkfläche selbst beteiligende Fraktur ist. Oftmals ist der Einblick in das Gelenk nicht möglich, jedoch sollten die Kirschner-Bohrdrähte so nahe wie möglich an, aber nicht in das Gelenk platziert werden, um den Femurkopf adäquat zu fixieren. Beim Einbringen der KirschnerBohrdrähte ist es durch die intraoperative Darstellung in den verschiedenen Ebenen der Situation mittels C-Bogen möglich, die Implantate präzise zu setzen (Abb. 10).

\section{Einsatz in der Neurochirurgie}

Für verschiedene neurochirurgische Eingriffe ist die intraoperative Bildgebung ebenfalls sehr hilfreich.

So kommen beim Hund und auch bei der Katze Bandscheibenvorfälle vor, die bei neurologischen Ausfällen oder persistierender Schmerzhaftigkeit eines operativen Eingriffs bedürfen. Die Diagnostik erfolgt neben der klinisch/neurologi- 
schen Untersuchung zur Eingrenzung der Lokalisation des Vorfalls optimalerweise mithilfe der Magnetresonanztomografie. Hierdurch kann nicht nur die genaue Lage des Vorfalls, sondern auch die Seitenbetonung präoperativ sehr gut und für den Zugang entscheidend dargestellt werden. Intraoperativ kann der betroffene Zwischenwirbelspalt bzw. die beiden angrenzenden Wirbelkörper zwar recht gut palpatorisch anhand z.B. der Dornfortsätze und anatomischer Landmarks identifiziert werden. Zur Absicherung ist es jedoch sehr hilfreich, die Lokalisation des Zwischenwirbelbereichs intraoperativ mit dem C-Bogen zu überprüfen. Auch bei anderen Eingriffen an der Wirbelsäule ist die intraoperative Bildgebung von Vorteil, wie z. B. bei Arachnoidzysten, zervikaler Instabilität, extramedullären, intraduralen Tumoren oder bei anderen Läsionen im Bereich der Wirbelsäule.

So kann unter Durchleuchtung der exakte Zwischenwirbelspalt oder der Wirbelkörper z. B. durch Einbringen einer Kanüle über der Lokalisation oder in den Dornfortsatz des Wirbels als Orientierungshilfe ermittelt werden (Abb. 11).

Auch bei Frakturen und Luxationen der Wirbelsäule wird die intraoperative Bildgebung in der Veterinärmedizin häufig eingesetzt. Traumatische Läsionen der Wirbelsäule kommen in allen Bereichen der Wirbelsäule vor und bedürfen in vielen Fällen einer chirurgischen Stabilisierung. Hier kommen neben der präoperativen Diagnostik im Hinblick auf eine Schädigung des Rückenmarks (CT, MRT) verschiedene chirurgische Verfahren zur Stabilisierung zum Einsatz. Wichtig ist hier die intraoperative Bildgebung zur Überprüfung der Positionierung der Implantate, denn der Korridor für das Einbringen von Schrauben oder Kirschner-Bohrdrähten ist sehr klein und eine weitere Schädigung des Rückenmarks kann durch den Einsatz des Bildwandlers vermieden werden. Beispiele sind hier das Einbringen von Platte und Schrauben (Abb.12) oder auch die interne Fixation mittels Kirschner-Bohrdrähten, die anschließend über Knochenzement (z.B. Palacos) miteinander verbunden werden.

\section{Einsatz bei kardiologischen Eingriffen}

Die intraoperative Bildgebung wird auch bei kardiologischen Eingriffen, wie beispielsweise der Implantation eines Herzschrittmachers [10], eingesetzt.
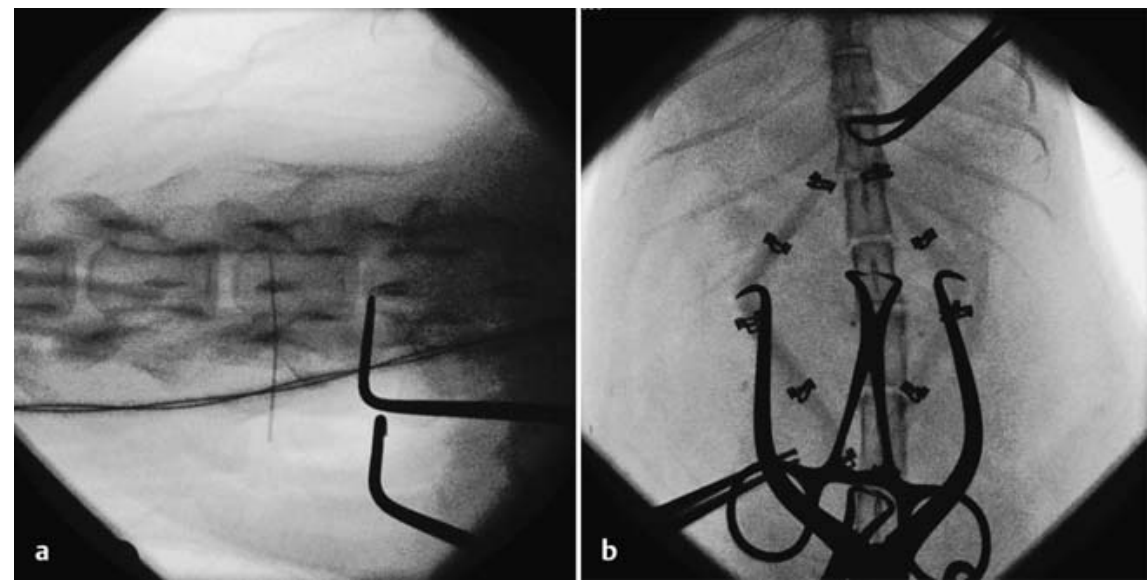

Abb.11a und b Intraoperative Bildgebung zur Lokalisierung eines Zwischenwirbelspalts bei einem Bandscheibenvorfall (a) bzw. einer Wirbelfraktur (b). Hier wird mit einer Kanüle (a) bzw. einer Backhaus-Klemme (b) die Position im Bereich des Dornfortsatzes markiert, um die genaue Lokalisation zu markieren. Als Besonderheit liegt im Bild links (a) eine Keilwirbelbildung im Bereich des Bandscheibenvorfalls vor.

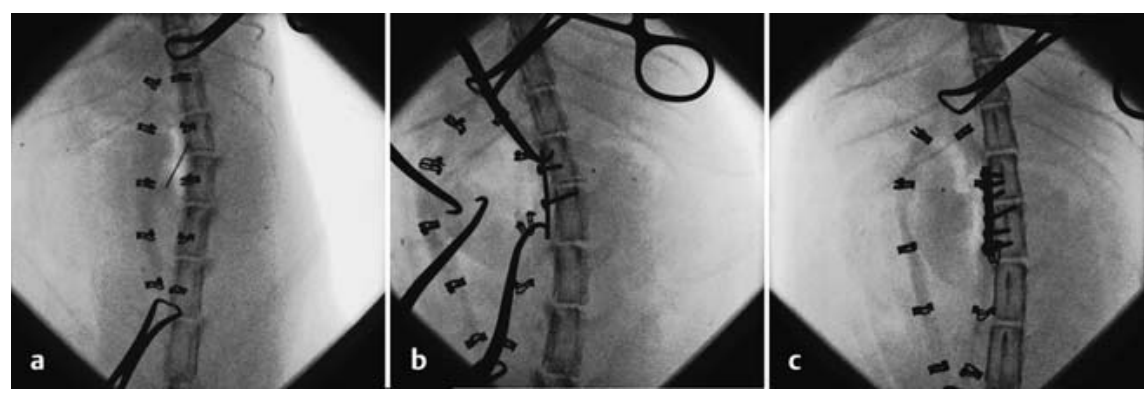

Abb. 12a bis c Fixation einer Luxation im Bereich der Lendenwirbelsäule. Hier wurde der kranial gelegene Wirbelkörper zunächst mithilfe einer Kanüle lokalisiert (a) und nach Reposition mittels einer winkelstabilen Platte und Schrauben fixiert. Nach Anbringen der Platte wurde zunächst die Lage der Platte und die Position der ersten Schrauben (b) unter Durchleuchtung überprüft und am Schluss die endgültige Fixation (c).

Die Implantation erfolgt i.d.R. über die rechte Jugularvene, durch die die Elektrode unter Durchleuchtungskontrolle in das Herz vorgeschoben wird. Nachdem die Funktion der Elektrode getestet wurde, wird sie nach kaudal getunnelt und an den Schrittmacher angeschlossen. Dieser wird dann subkutan in einer kleinen Tasche implantiert [10]. Auch bei anderen gefäßchirurgischen Eingriffen, wie z.B. einem Vorhofseptumdefekt [11] oder einem persistierenden Ductus arteriosus botalli $[12,13]$, hat die intraoperative Bildgebung eine große Bedeutung und ist eine erhebliche Erleichterung. Ohne intraoperative Bildgebung wäre dieser Eingriff nicht machbar, wobei zur Darstellung der entsprechenden Bereiche immer die Verwendung von Kontrastmittel erforderlich ist.

Der persistierende Ductus arteriosus botalli (PDA) ist eine der häufigsten kongenitalen Herzerkrankungen, insbesondere beim Hund, wobei es sich um eine persitierende Verbindung zwischen der Aorta und der Pulmonalarterie handelt. Normalerweise verschließt sich diese Verbindung nach der Geburt spontan. Bleibt sie jedoch bestehen, führt das zu einer $\mathrm{zu}$ starken Lungendurchblutung und letztendlich $\mathrm{zu}$ einer Überlastung des Herzens [14]. Daher muss diese Verbindung operativ durch einen Verschluss mittels eines Coils [15] oder eines Amplatzers verschlossen werden. Der Verschluss eines PDAs mit dem Amplatzer ${ }^{\circledR}$ Duct Occluder ist heute ein Standardverfahren. In München wird es von der kardiologischen Abteilung der Medizinischen Kleintierklinik der LMU routinemäßig durchgeführt. Über einen speziellen Katheter wird der Amplatzer über die Femoralarterie in die Aorta bis zum Herzen vorgeschoben und nach fluoroskopischer Darstellung des PDAs dort abgesetzt. Der vollständige Verschluss des PDAs wird mithilfe von Kontrastmittel kontrolliert (Abb. 13) 

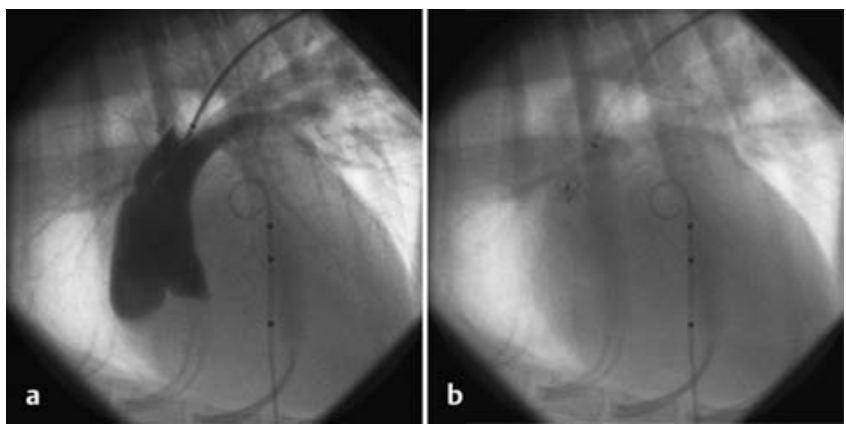

Abb. $13 \mathrm{a}$ und $\mathrm{b}$ Intraoperative Bildgebung nach Setzen eines Amplatzers bei Vorliegen eines PDAs (a). Mithilfe einer Kontrastdarstellung wird der vollständige Verschluss des PDAs kontrolliert (b).
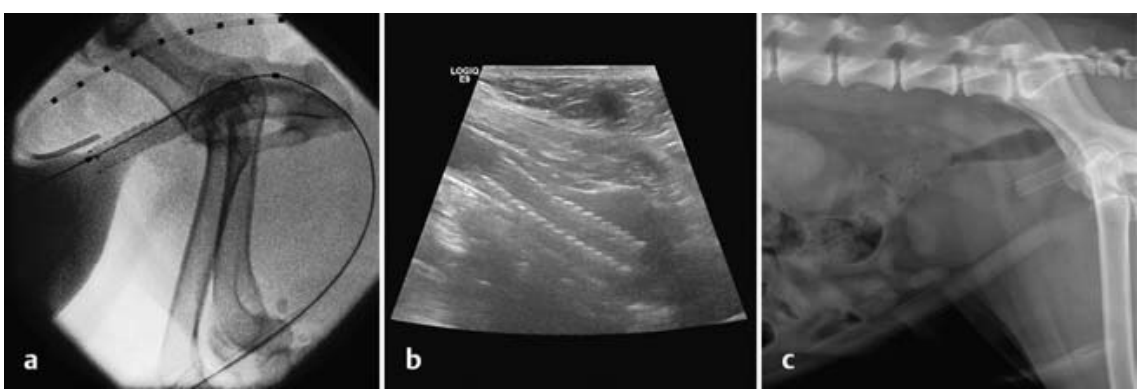

Abb. 14a bis c Intraoperative Darstellung mittels C-Bogen (a) bzw. Ultraschalluntersuchung (b) eines Urethrastents, welcher aufgrund eines Übergangszellkarzinoms der Urethra am Übergang zur Blase gelegt wurde. Die anschließende Röntgenkontrolle zeigt den optimalen Sitz des Stents am Übergang der Urethra zum Blasenhals.

\section{Einsatz in der Urologie}

In der Urologie wird die intraoperative Bildgebung sowohl für Erkrankungen des Ureters als auch der Urethra, insbesondere für das Einsetzen von Stents, genutzt.

Indikationen für die Implantation eines Stents in die Urethra, liegen i.d.R. nur bei männlichen Hunden vor, wobei es sich hier um Strikturen oder das Vorliegen von Obstruktionen z.B. durch Übergangszellkarzinome handelt $[16,17]$. Letzteres dient als palliative Maßnahme und wird i.d.R. begleitet von einer Chemotherapie des Hundes. Nach Stellung der Diagnose mittels Sonografie, Röntgenkontrastdarstellung und/oder ggf. Urethroskopie erfolgt die Implantation des Urethrastents unter Einsatz des C-Bogens. Hiermit kann einerseits die Größe und Länge des Stents evaluiert wie auch die korrekte Platzierung kontrolliert werden (Abb. 14a-c).

Neben dem Einsatz des C-Bogens bei Legen von intraluminalen Stents in die Urethra wird er auch als bildgebende intraoperative Darstellung im Rahmen der Therapie von ektopischen Ureteren eingesetzt. Beim Hund kommen ektopische Ureteren bei verschiedenen Hunderassen vor und es gibt Rassendispositionen, z. B. beim Husky oder Golden Retriever.
Meist sind weibliche Tiere betroffen, aber sie kommen auch bei Rüden vor. Das Leitsymptom bei Vorliegen eines ektopischen Ureters ist die Harninkontinenz. Meist wird die Erkrankung beim Welpen diagnostiziert und sie kann einoder beidseitig vorkommen. Die Diagnose wird i.d.R. mithilfe der Röntgenkontrastuntersuchung, dem Ultraschall, der computertomografischen oder der endoskopischen Untersuchung gestellt [18]. Oft geht die Erkrankung mit einer Dilatation des betroffenen Ureters einher, was sich sehr gut mithilfe der Ultraschalluntersuchung darstellen lässt. In unverändertem Zustand ist der Ureter selbst jedoch nicht darstellbar. Häufig (ca. 90\%) kommt der Ureter von der Niere anatomisch korrekt an der Blasenwand (Trigonum vesicae) an, verläuft dann jedoch tunnelartig in der Submucosa der Blasenwand (intramural) bis zu seiner ektopischen Öffnung in der Urethra. Oft liegt die Öffnung relativ weit in der Urethra und lässt sich nicht direkt mithilfe des Ultraschalls oder der Computertomografie darstellen. Die genaue Lokalisation der Öffnung kann dann mithilfe der Endoskopie dargestellt werden. In diesen Fällen kann nach Zystotomie der intramurale Verlauf des Ureters durch Kompression im distalen Bereich und nachfolgender Stauung desselben sichtbar gemacht werden [19]. Die Eröffnung erfolgt dann durch eine ca. 0,5-
$1 \mathrm{~cm}$ lange Inzision durch die Blasenschleimhaut in den Ureter. Anschließend wird die Schleimhaut des Ureters mit der Blasenschleimhaut vernäht (Ureterozystoneostomie). Als Nahtmaterial wird ein dünner, atraumatischer, resorbierbarer monofiler Faden der Stärke 6/0 oder $7 / 0$ verwendet (PDS). In einigen Zentren wird seit einiger Zeit zur Behandlung eines intramural verlaufenden ektopischen Ureters bei der Hündin eine Laserablation mithilfe eines Diodenlasers unter endoskopischer und fluoroskopischer Kontrolle angewendet [18, 20]. Hier wird die intraoperative Bildgebung zur genauen Lokalisation und Verlaufsdarstellung des ektopischen Ureters eingesetzt.

In manchen Fällen (ca. 10\%) mündet der Ureter nicht im Bereich des Trigonum vesicae und verläuft intramural, sondern läuft direkt in Richtung Urethra oder auch Uterus/Vestibulum vaginae und mündet hier. Dies kann ebenfalls unter fluoroskopischer Kontrolle dargestellt werden (Abb. 15). Hier kann die Therapie jedoch nicht durch eine Laserbehandlung erfolgen, sondern der Ureter muss dann nach Laparotomie an seiner Insertionsstelle abgesetzt und anschließend an die Dorsalfläche der Harnblase neu re-inseriert (Neoureterozystostomie) werden $[18,19]$.

Eine andere Indikation für die intraoperative Bildgebung ist das Vorliegen von Uretersteinen oder Ureterstrikturen bei der Katze [21]. Hier wird ein Ureterstent unter fluoroskopischer Kontrolle unter Zuhilfenahme von Kontrastmittel vom Nierenbecken über die Striktur bzw. den Ureterstein geschoben und in der Blase platziert. Auch beim Hund wird das Legen eines Ureterstents zur Therapie einer bilateralen kongenitalen Ureterstriktur unter Zuhilfenahme der intraoperativen Fluoroskopie mit Kontrastmittel beschrieben [22].

\section{Einsatz des C-Bogens bei weiteren Indikationen}

Immer häufiger kommt die intraoperative Bildgebung für die Versorgung von angeborenen portosystemischen Shunts zum Einsatz.

Bei einem portosystemischen Shunt handelt es sich um einen Kurzschluss der abführenden Gefäße des MagenDarm-Kanals meist mit der V. cava caudalis. Dadurch wird die Leber umgangen und das Blut aus dem Einzugsgebiet wird 


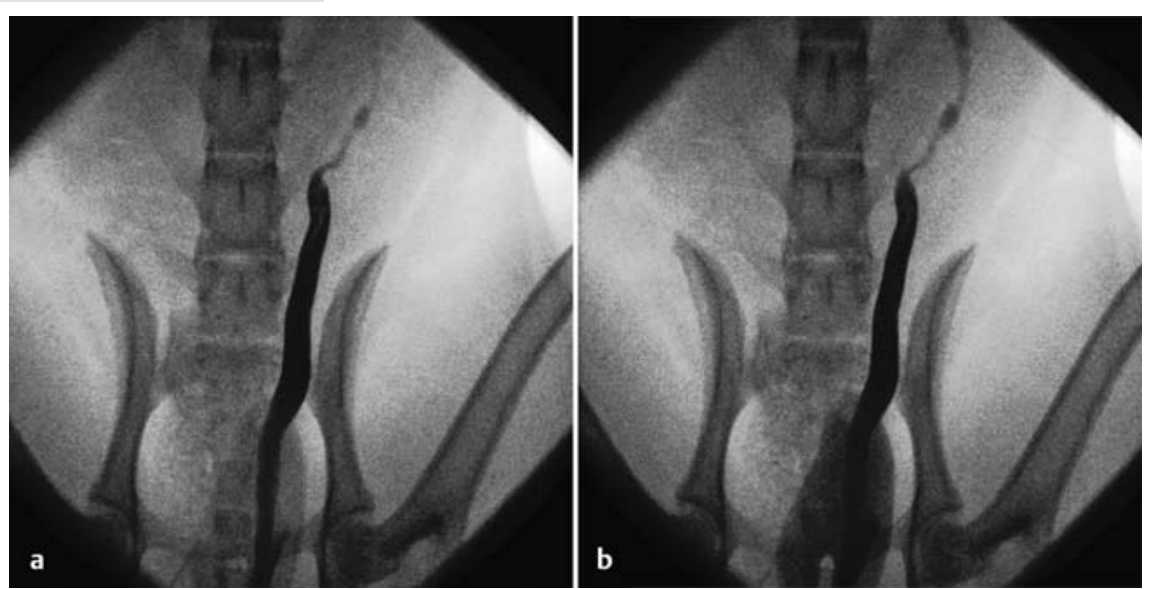

Abb. 15a und b Intraoperative fluoroskopische Darstellung eines ektopischen Ureters. Der ektopische Ureter ist deutlich mit Kontrastmittel gefüllt und die Mündung liegt im Bereich des Vestibulum vaginae (a). Durch weitere Konstrastmittelapplikation zeigt sich auch eine Füllung des Vestibulums (b). In diesem Fall kann keine Laserablation des ektopischen Ureters erfolgen, da er nicht intramural in der Blasenwand verläuft.
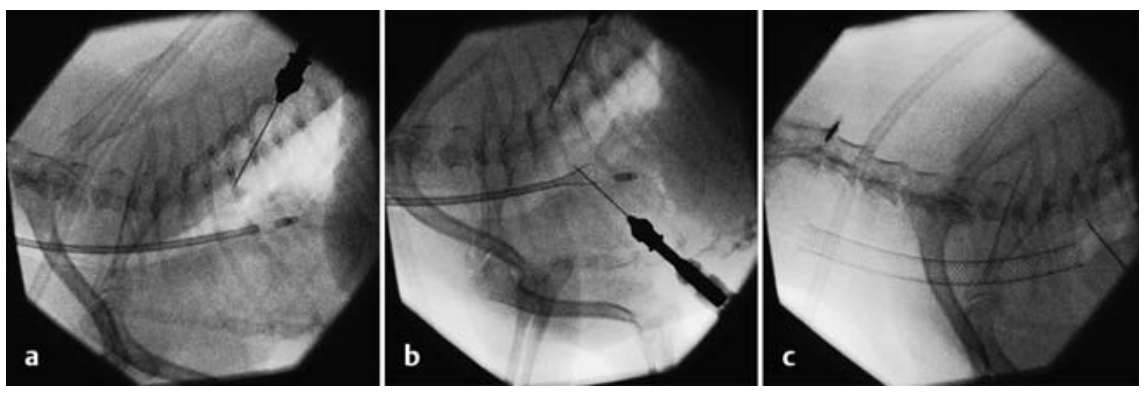

Abb. 16a bis c Intraoperative Bildgebung bei der Implantation eines intraluminalen trachealen Stents. Der Führungskatheter wird unter Kontrolle mit dem C-Bogen in die Trachea eingeführt (a), bei korrekter Position, die mithilfe von Kanülen markiert werden kann, wird der Stent geöffnet (b) und weitet am Ende der Prozedur und Entfernung des Führungskatheters die Trachea und reicht in diesem Fall über die gesamte Länge der Trachea (c).

nicht über die Pfortader in die Leber geleitet. Ein portosystemischer Shunt kann entweder innerhalb der Leber (intrahepatischer Shunt) oder außerhalb der Leber (extrahepatischer Shunt) lokalisiert sein. Da es sich meistens um eine angeborene Erkrankung handelt, werden die meisten Hunde mit unter $1 \mathrm{Jahr}$ dem Tierarzt vorgestellt. Hinweise auf das Vorliegen eines Shunts geben neben den klinischen Symptomen Veränderungen der Laborwerte. Einen wichtigen Hinweis liefern die bei den meisten Patienten erhöhten Ammoniakwerte. Bei Shuntverdacht wird heute neben dem Ultraschall die Kernspintomografie (MRT) oder die Computertomografie (CT) zur Diagnostik genutzt. Mit beiden Verfahren kann das Shuntgefäß 3-dimensional dargestellt werden, was dem Chirurgen in seiner OP-Planung hilft. Die Therapie der Wahl ist der möglichst frühzeitige Verschluss des Shuntgefäßes, welches in den meisten Fällen über eine Laparotomie erfolgt. Insbesondere bei in- trahepatischen Shuntgefäßen, bei denen der operative Eingriff nicht ganz trivial ist und viel Erfahrung des Chirurgen erfordert, wird in einigen Zentren zum Verschluss des Shuntgefäßes auch die minimalinvasive Therapie mittels transvenöser Coil-Embolisation [23, 24] oder durch eine Kombination eines Stents mit einer Coil-Embolisation [25] durchgeführt. Bei der Coil-Embolisation wird, wie beim Verschluss eines PDAs, das Shuntgefäß unter fluoroskopischer Darstellung aufgesucht und es wird an der Mündung unter Kontrolle mit dem C- Bogen ein Coil platziert (Abb. 16). Bei der anderen Technik wird zunächst das Gefäß aufgesucht, in das der Shunt mündet und es wird in diesem Bereich mit einem Stent geschient. Anschließend werden kleine Coils durch die Lücken des Stents in die Shuntgefäßeintrittsstelle eingebracht, sodass es im weiteren Verlauf zu einer Thrombenbildung im Bereich der Gefäßmündung kommt, wodurch der Verschluss des Shuntgefäßes erzielt wird.
Auch an anderen Lokalisationen werden intraluminale Stents eingesetzt, bei denen der Einsatz der intraoperativen Bildgebung erforderlich ist. So gibt es insbesondere bei kleinen Hunderassen, wie z.B. dem Yorkshire Terrier, eine Erkrankung der Atemwege, die mit einem Kollaps der Trachea einhergeht. Die klinischen Symptome zeigen sich in einer zunehmenden Dyspnoe, die sich insbesondere bei Anstrengung oder in schweren Fällen auch im Ruhezustand zeigt. Die Diagnose wird mit der Röntgenuntersuchung, ggf. auch unter Durchleuchtung, gestellt.

Die Therapie erfolgt durch das Legen eines intraluminalen Trachealstents, der in Allgemeinanästhesie unter Kontrolle mit einem C-Bogen implantiert wird $[26,27]$.

Bei den Stents werden i.d.R. Nitinolstents (Nickel-Titan-Legierung) verwendet [27]. Der Stent wird nach endoskopischer Bestätigung der Diagnose unter fluorokopischer Kontrolle mit einem speziellen Führungskatheter in die Trachea eingeführt (Abb. 16) und die Länge und Lage mit dem C-Bogen überprüft. Bei korrekter Position wird der in dem Katheter liegende Stent freigegeben und expandiert innerhalb der Trachea, um so die Luftwege offen zu halten.

\section{Intraoperative Bildgebung mittels anderer bildgebender Verfahren}

\section{Intraoperative Bildgebung mittels Ultraschall}

Die Bildgebung mittels Ultraschall findet intraoperativ bspw. bei der Operation eines intrahepatischen Shunts Anwendung als Alternative zur Portografie. Mithilfe des Duplex-Dopplers kann man quantitativ portale hämodynamische Unterschiede identifizieren. Die intraoperative B-Mode-Ultraschalldiagnostik ermöglicht eine präzise Information als Momentaufnahme über die Lage des Shunts, der Portalvenen und der Gefäße in der Umgebung, was für die Operation relevant ist [28].

Auch bei Bandscheibenvorfällen kann der intraoperative Ultraschall eingesetzt werden [29]. Bei einer Frequenz von 5$10 \mathrm{MHz}$ ist es neben der Darstellung von Bandscheibenmaterial möglich, spinale Strukturen wie Dura mater, Pia mater, Subarachnoidalräume, Zentralkanal und Rückenmark sichtbar zu machen. 
Einsatz von intraoperativer Computertomografie (CT) und Magnetresonanztomografie (MRT)

In der Veterinärmedizin spielt die intraoperative Computertomografie eine Rolle bei der Planung und Durchführung von diagnostischen Biopsieentnahmen und Feinnadelaspirationen im Kopfbereich oder bei intrathorakalen Läsionen. Komplikationen wie Pneumothorax oder pulmonale Blutungen können so rasch erkannt werden. CT-gestützte Probeentnahmen sind relativ sicher und hilfreich bei der Diagnose intrathorakaler Umfangsvermehrungen, Läsionen oder Neoplasien. Auch in der Neuronavigation durch präzise, CT-gestützte, 3-dimensionale Bilder wird auch in der Veterinärmedizin dieses bildgebende Verfahren intraoperativ angewendet [30,31]. Bei gefäßchirurgischen Eingriffen, bspw. der chirurgischen Versorgung bei einem Aneurysma, kann die Magnetresonanztomografie (MRT) intraoperativ hilfreich sein. Hiermit können Aussagen über die Position eines Katheters und von Führungsschienen getroffen werden [32]. Jedoch ist diese Indikation selten und die intraoperative MRT wird in der Veterinärmedizin insgesamt nur sehr selten angewendet.

\section{Literatur}

${ }^{1}$ SIEMENS. Gebrauchsanweisung für ARCADIS Varic, Technische Daten, SPR2310.620.01.03.01, gedruckt in der Bundesrepublik Deutschland 02/06

${ }^{2}$ Djouchadar D. Röntgenhelfer Chirurgie - Ein Leitfaden für den C-Bogeneinsatz im Operationssaal. Berlin: Bengelsdorf \& Schimmel; 2002: 19

${ }^{3}$ Wilde P, Schmidt-Horlohé K, Reimertz C et al. Stellenwert der intraoperativen 3D-Bildgebung bei der Versorgung intraartikulärer Frakturen an OSG und USG. In: Meeting Abstract. Deutscher Kongress für Orthopädie und Unfallchirurgie 21.-24.10.2009, Berlin. Düsseldorf: GMS Publishing House; 2009: DocWI45-1192

${ }^{4}$ Franke J, von Recum J, Wendl K et al. Intraoperative dreidimensionale Bildgebung - nützlich oder notwendig? Unfallchirurg, 2013; 116: $185-190$

${ }^{5}$ Richter M. Warum zweimal operieren, wenn es eine intraoperative 3D-Bildgebung gibt. Inside: Health, Dezember 2012: 24-25. Magazin von Siemens für Entscheider im $\mathrm{Ge}$ sundheitswesen, www.healthcare.siemens. de

${ }^{6}$ Meyer-Lindenberg A. Planung, Vorbereitung und Kontrolle bei Eingriffen in der Veterinärmedizin. OP-Journal, 2011; 27: 90-98
7 Meyer-Lindenberg A, Fehr M, Nolte I. Shortand long-term results after surgical treatment of an ununited anconeal process in the dog. Vet Comp Orthop Traumatol 2001; 14: 101-110

8 Cook LJ, Tomlinson JL, Reed AL. Fluoroscopically guided closed reduction and internal fixation of fractures of the lateral portion of the humeral condyle: prospective clinical study of the technique and results in ten dogs. Vet Surg 1999; 28: 315-321

${ }^{9}$ Leasure CS, Lewis DD, Sereda CW et al. Limited open reduction and stabilization of sacroiliac fracture-luxations using fluoroscopically assisted placement of a trans-iliosacral rod in five dogs. Vet Surg 2007; 36: 633-643

10 Yin L, Laske TG, Rakow N et al. Intracardiac echocardiography-guided his bundle pacing and atrioventricular nodal ablation. Pacing Clin Electrophysiol 2008; 31: 536-542

11 Gordon SG, Miller MW, Roland RM et al. Transcatheter atrial septal defect closure with the Amplatzer atrial septal occluder in 13 dogs: short- and mid-term outcome. J Vet Intern Med 2009; 23: 995-1002

12 Markovic LE, Kellihan HB, Roldán-Alzate A et al. Advanced multimodality imaging of an anomalous vessel between the ascending aorta and main pulmonary artery in a dog. J Vet Cardiol 2014; 16: 59-65

${ }^{13}$ Hildebrandt N, Schneider C, Schweigl T et al. Long-term follow-up after transvenous single coil embolization of patent ductus arteriosus in dogs. J Vet Intern Med 2010; 24 : 1400-1406

${ }^{14}$ Saunders AB, Gordon SG, Boggess MM et al. Long-term outcome in dogs with patent ductus arteriosus: 520 cases (1994-2009). J Vet Intern Med 2014; 28: 401-410

15 Singh MK, Kittleson MD, Kass PH et al. Occlusion devices and approaches in canine patent ductus arteriosus: comparison of outcomes. J Vet Intern Med 2012; 26: 85-92

${ }^{16}$ McMillan SK, Knapp DW, Ramos-Vara JA et al. Outcome of urethral stent placement for management of urethral obstruction secondary to transitional cell carcinoma in dogs: 19 cases (2007-2010). J Am Vet Med Assoc 2012: 241: 1627-1632

17 Blackburn AL, Berent AC, Weisse CW et al. Evaluation of outcome following urethral stent placement for the treatment of obstructive carcinoma of the urethra in dogs: 42 cases (2004-2008). J Am Vet Med Assoc 2013; 242: 59-68

${ }^{18}$ Davidson AP, Westropp JL. Diagnosis and management of urinary ectopia. Vet Clin North Am Small Anim Pract 2014; 44: 343-353

${ }^{19}$ Reichler IM, Eckrich Specker C, Hubler M et al. Ectopic ureters in dogs: clinical features, surgical techniques and outcome. Vet Surg 2012; 41: 515-522

20 Berent AC, Weisse CW, Mayhew PD et al. Evaluation of cystoscopic-guided laser ablation of intramural ectopic ureters in female dogs. J Am Vet Med Assoc 2012; 240: 716-725

21 Berent AC, Weisse CW, Todd K et al. Technical and clinical outcomes of ureteral stenting in cats with benign ureteral obstruction: 69 cases (2006-2010). J Am Vet Med Assoc 2014; 244: 559-576

22 Lam NK, Berent AC, Weisse CW et al. Endoscopic placement of ureteral stents for treat- ment of congenital bilateral ureteral stenosis in a dog. J Am Vet Med Assoc 2012; 240: $983-$ 990

${ }^{23}$ Asano K, Watari T, Kuwabara M et al. Successful treatment by percutaneous transvenous coil embolization in a small-breed dog with intrahepatic portosystemic shunt. J Vet Med Sci 2003; 65: 1269-1272

${ }^{24}$ Weisse C, Berent AC, Todd Ket al. Endovascular evaluation and treatment of intrahepatic portosystemic shunts in dogs: 100 cases (2001-2011). J Am Vet Med Assoc 2014; 244: 78-94

25 Schneider M, Plassmann M, Rauber K. Intrahepatic venous collaterals preventing successful stent-supported coil embolization of intrahepatic shunts in dogs. Vet Radiol Ultrasound 2009; 50: 376-384

${ }^{26}$ Beal MW. Tracheal stent placement for the emergency management of tracheal collapse in dogs. Top Companion Anim Med 2013; 28: 106-111

${ }^{27}$ Beranek J, Jaresova H, Rytz U. Use of nitinol self-expandable stents in 26 dogs with tracheal collapse. Schweiz Arch Tierheilkd 2014; 156: 91-98

${ }^{28}$ Szatmári V, van Sluijs FJ, Rothuizen $J$ et al. Intraoperative ultrasonography of the portal vein during attenuation of intrahepatic portocaval shunts in dogs. J Am Vet Med Assoc 2003; 222: 1086-1092

${ }^{29}$ Kramer M, Golla T, Schmidt MJ. [Intraoperative sonography for examination of intervertebral disk extrusion in the dog]. Tierarztl Prax Ausg K Kleintiere Heimtiere 2011; 39: 397-403

${ }^{30}$ Zekas LJ, Crawford JT, O'Brien RT. Computed tomography-guided fine-needle aspirate and tissue-core biopsy of intrathoracic lesions in thirty dogs and cats. Vet Radiol Ultrasound 2005; 46: 200-204

31 Taylor AR, Cohen ND, Fletcher S et al. Application and machine accuracy of a new frameless computed tomography-guided stereotactic brain biopsy system in dogs. Vet Radiol Ultrasound 2013; 54: 332-342

32 Strother CM, Unal I, Frayne R et al. Endovascular treatment of experimental canine aneurysms: feasibility with MR imaging guidance. Radiology 2000; 215: 516-519

\section{Dr. med. vet. Tim Kilchling}

Wissenschaftlicher Mitarbeiter

Prof. Dr. med. vet.

Andrea Meyer-Lindenberg

Vorstand der Chirurgischen und

Gynäkologischen Kleintierklinik

Chirurgische und Gynäkologische Kleintierklinik

Ludwig-Maximilians-Universität

München

Veterinärstraße 13

80539 München

meyer-lindenberg@

chir.vetmed.uni-muenchen.de 